\title{
Synthetic studies on the preparation of nucleoside 5'-H-phosphonate monoesters under the Mitsunobu reaction conditions
}

\author{
Gaston Lavéna and Jacek Stawinskia,b* \\ a Department of Organic Chemistry, Arrhenius Laboratory, Stockholm University,S-10691

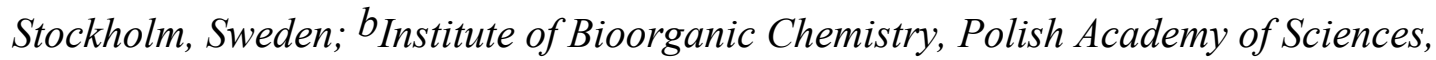 \\ Noskowskiego 12/14, 61-704 Poznan, Poland \\ E-mail: js@organ.su.se
}

\section{Dedicated to Professor Harry Lönnberg on the occasion of his 60th birthday}

\begin{abstract}
A reaction of suitably protected nucleosides with phosphonic acid in the presence of diethyl azodicarboxylate and triphenylphosphine in pyridine provided in good yields the corresponding 5'-H-phosphonate monoesters.
\end{abstract}

Keywords: H-Phosphonate monoesters, phosphonic acid, the Mitsunobu reaction

\section{Introduction}

The Mitsunobu reaction is a versatile and widely used method in organic synthesis because of its scope, stereospecificity, and mild experimental conditions. ${ }^{1,2}$ Typically, it involves the condensation of an acidic pronucleophile ( $\mathrm{HNu}$, e.g. carboxylic acids, phenols, imides, etc.) and an alcohol (ROH), promoted by triphenylphosphine (TPP) and diethyl azodicarboxylate (DEAD), to afford the product $\mathrm{RNu}$, along with diethoxycarbonylhydrazine and triphenylphosphine oxide. This redox mediated condensation is frequently used for the formation of $\mathrm{C}-\mathrm{O}, \mathrm{C}-\mathrm{N}, \mathrm{C}-\mathrm{S}$, or $\mathrm{C}-\mathrm{C}$ bonds in a stereospecific manner, in the presence of a wide range of functional groups. ${ }^{1}$ Due to known separation problems, many recent efforts in the Mitsunobu reaction research have been directed toward modifying triphenylphosphine and azodicarboxylate reagents $^{3,4}$ to facilitate purification procedures, or to develop special, usually chromatographyfree, separation strategies. ${ }^{4,5}$

As part of our program in developing synthetic methods for biologically important phosphates and their analogues based on H-phosphonate chemistry, ${ }^{6,7}$ we became interested in the Mitsunobu reaction for two reasons. Firstly, it permits a condensation to be carried out under mild, virtually neutral conditions, and secondly, it involves activation of a hydroxyl function of an alcohol, rather than conversion of a pronucleophile into a reactive species. The latter aspect 
seemed particularly appealing for a stereospecific synthesis of phosphate analogues using Pchiral phosphorus precursors, ${ }^{7,8}$ as it would alleviate a potential problem of epimerization at the phosphorus center.

Owing to the importance and widespread use of the Mitsunobu reaction, its mechanism have been extensively investigated. ${ }^{1,9,10}$ Although, inversion of configuration in the hydroxylic component is typically observed, ${ }^{1}$ the mechanism turned out to be rather complex. The first step of the reaction is an irreversible addition of TPP to DEAD to form the Morrison-Brunn-Huisgen betaine, ${ }^{11}$ which may cascade into a product (or by-products) in several ways, depending on reactivity of the alcohol and a pKa value of the acidic pronucleophile used. ${ }^{12}$ Recent studies on lactonization of sterically hindered alcohols showed that the Mitsunobu reaction can occur with retention of configuration, ${ }^{10}$ and the density functional investigations ${ }^{13}$ supported the view that the Mitsunobu reaction is fundamentally capable to affording products either with inversion or retention of configuration. Thus, Mitsunobu type of reactions, particularly those involving new types of pronucleophiles, have to be carefully scrutinized for a mechanism operating.

Concerning preparation of phosphates or their analogues, Mitsunobu et al. ${ }^{14,15}$ used TPP and DEAD as an activating system for phosphorylation of nucleosides with dibenzyl phosphate. The reaction gave acceptable yields of pyrimidine nucleoside phosphates (40-70\%), but completely failed for adenosine and guanosine. More recently, the Mitsunobu reaction conditions were investigated for the purpose of C-phosphonate synthesis using methyl- and benzylphosphonate monoesters. ${ }^{16}$ Although a variety of acidic pronucleophiles have been investigated, ${ }^{1}$ there has been no report, to our knowledge, on the formation of H-phosphonate mono- and H-phosphonate diesters via the Mitsunobu coupling reaction.

In this paper we present our preliminary synthetic investigation on the preparation of nucleoside 5'-H-phosphonates under the Mitsunobu reaction conditions, and some ${ }^{31} \mathrm{P}$ NMR studies on the formation of H-phosphonate diesters in the presence of DEAD and TPP.

\section{Results and Discussion}

To check a possibility of formation of $\mathrm{H}$-phosphonate monoesters under the Mitsunobu reaction conditions, we reacted $3^{\prime}-O$-protected thymidine derivative 1a as a model $1^{\circ}$ alcohol with phosphonic acid in the presence of DEAD and TPP under various experimental conditions. The reaction was very sensitive, in terms of the reaction time and purity of the product formed $\left({ }^{31} \mathrm{P}\right.$ NMR spectroscopy analysis), to the ratio of the reactants, solvents, and the bases present. The best results were obtained when phosphonylation of nucleoside 1a was carried out in pyridine in the presence of 2 equiv. each of DEAD and TPP, and by using 2 equiv. of phosphonic acid (Scheme 1). These conditions secured a quantitative formation of the desired nucleoside $5^{\prime}-\mathrm{H}-$ phosphonate $\mathbf{2 a}$ within ca $20 \mathrm{~min}\left({ }^{31} \mathrm{P}\right.$ NMR analysis), and on a preparative run, compound $\mathbf{2 a}$ was isolated in $85 \%$ as a triethylammonium salt. 


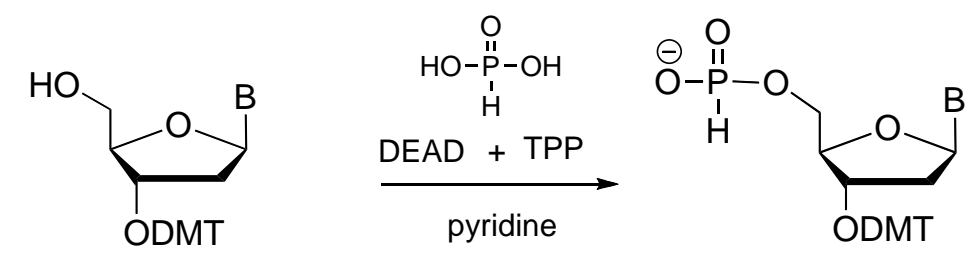

1a-d

2a-d
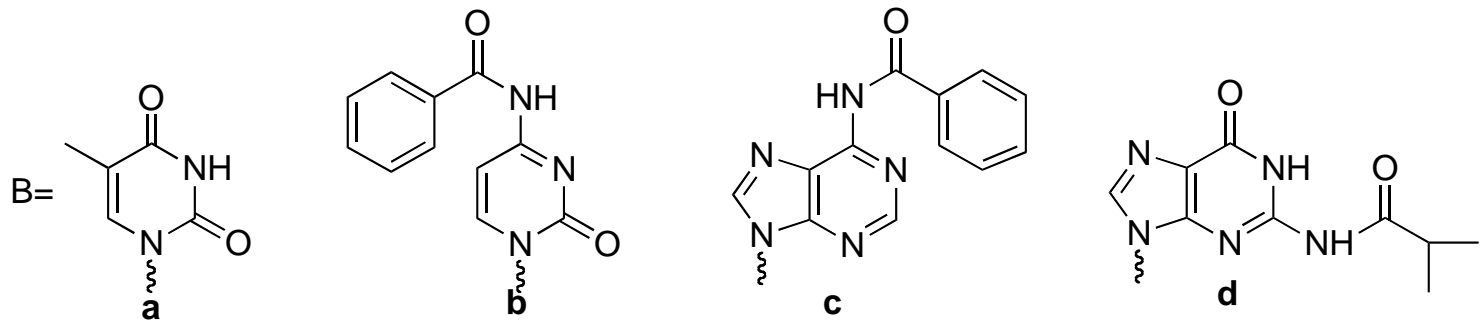

DMT = 4,4'-Dimethoxytrityl; DEAD = diethyl azodicaroxylate; TPP = triphenylphosphine

\section{Scheme 1}

The above protocol was successfully applied to the other common nucleosides, namely to cytidine (1b), adenosine (1c) and guanosine (1d), derivatives, to produce the corresponding 5'H-phosphonate monoesters 2 b-d in good isolated yields (74-84\%).

One should emphasise here a relatively high yield of phosphonylation of adenosine derivative 1c (74\%) under the developed reaction conditions. Purine nucleosides, and adenosine in particular, are known to be highly susceptible to intramolecular cyclization forming, via a nucleophilic attack of nitrogen $\mathrm{N}-3$ of the purine base on the $5^{\prime}$-carbon atom, $N^{3}, 5^{\prime}$ cyclonucleosides, when the $5^{\prime}-\mathrm{OH}$ function is converted into a good living group. ${ }^{15,17}$ For this reason, usually only pyrimidine nucleosides can be phosphorylated (or acylated) under the Mitsunobu reaction conditions, while purine nucleosides either cannot be used as substrates ${ }^{14,15}$ or give mediocre yields. ${ }^{16}$

Although the Mitsunobu reaction of alcohols with carboxylic acids is assumed to proceed with the intermediacy of the corresponding alkoxyphosphonium salts, it is well documented that also carboxylic acid anhydrides can be formed under the reaction conditions. ${ }^{18}$ Since $\mathrm{H}-$ pyrophosphonate in pyridine is known to act as a phosphonylating agent for nucleosides, ${ }^{19}$ we could not exclude a possibility that this can be a reactive species involved in the reaction investigated. To verify this assumption, H-pyrophosphonate (generated in situ from phosphonic acid and pivaloyl chloride in pyridine $)^{19}$ was allowed to react with nucleoside $1 \mathrm{a}$ in pyridine. Unfortunately, the phosphonylation of 1a was significantly slower (50\% conversion after $4 \mathrm{~h}$ ) than that under the Mitsunobu conditions, and thus it is unlikely that phosphonylation of nucleosides with phosphonic acid in the presence of DEAD and TPP involved as a kinetically significant intermediate, H-pyrophosphonate. 
When the phosphonylation of nucleoside 1a under the Mitsunobu conditions (Scheme 1) was carried out in the presence of triethylamine, the reaction became significantly slower (few hours for the completion), and for very strong bases, such as 1,8-diazabicyclo[5.4.0]undec-7-ene (DBU), no product formation was observed even after prolonged reaction time (overnight). These experiments are consistent with the observed dependency of the Mitsunobu reaction on a pKa value of the acidic pronucleophiles, ${ }^{12}$ (in our case, acidities of the conjugated acids) and may point to the importance of protonation of the Morrison-Brunn-Huisgen betaine. ${ }^{11}$

\section{${ }^{31} \mathrm{P}$ NMR experiments on the formation of $\mathrm{H}$-phosphonate diesters}

We carried out also some preliminary ${ }^{31} \mathrm{P}$ NMR experiments on the formation of H-phosphonate diesters under the Mitsunobu coupling conditions (Scheme 2).

To this end we reacted H-phosphonate monoesters 3a,b (triethylammonium salt, $0.1 \mathrm{mmol}$ ) with nucleoside $4 \mathbf{b}(0.15 \mathrm{mmol})$ in the presence of DEAD $(0.15 \mathrm{mmol})$ and TPP $(0.15 \mathrm{mmol})$ in tetrahydrofuran $(2 \mathrm{~mL})$. The reactions were clean, went to completion within $2 \mathrm{~h}$, and the produced H-phosphonate diesters $\mathbf{5 b}$ and $\mathbf{5 d}$, respectively, were identical $\left({ }^{31} \mathrm{P} \mathrm{NMR}\right.$ data) to those prepared form H-phosphonates $\mathbf{3}$ and alcohols $\mathbf{4}$ using pivaloyl chloride as a condensing agent. $^{20}$ For the analogous reactions with ethanol, larger excess of DEAD, TPP and the alcohol ( 3 equiv. each) had to be used, apparently due to competing side reactions of the activated ethanol. In contrast to phosphonylation of nucleosides 1 with phosphonic acid (Scheme 1), the condensations of H-phosphonate monoesters $\mathbf{3}$ depicted in Scheme 2 did not work well in pyridine. For reasons to be identified yet, all the reactions in Scheme 2 proceeded only to $50 \%$ completion in pyridine, even after prolonged reaction time (overnight).

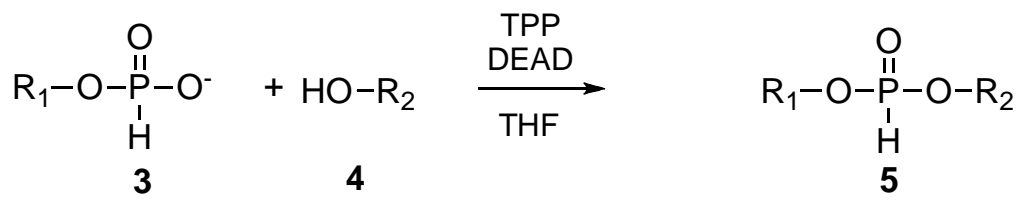
3a, $\mathrm{R}_{1}=$ ethyl
3b, $R_{1}=5^{\prime}$-O-dimethoxytritylthymidin-3'-yl
$4 a, R_{2}=$ ethyl
4b, $\mathrm{R}_{2}=3^{\prime}$-O-dimethoxytritylthymidin-5'-yl
$5 a, R_{1}=R_{2}=$ ethyl

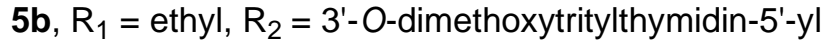
5c, $\mathrm{R}_{1}=5^{\prime}-\mathrm{O}$-dimethoxytritylthymidin-3'-yl, $\mathrm{R}_{2}=$ ethyl
5d, $R_{1}=5 '-O-d i m e t h o x y t r i t y l t h y m i d i n-3 '-y l, R_{2}=3 '-O-d i m e t h o x y t r i t y l t h y m i d i n-5 '-y l$

\section{Scheme 2}

In conclusion, we developed an efficient protocol for the synthesis of nucleoside 5'-Hphosphonate monoesters by reacting suitably protected nucleosides with triethylammonium salt of H-phosphonic acid in pyridine under the Mitsunobu reaction conditions. The method works 
well both for pyrimidine and purine nucleosides and can be considered and as an alternative procedure ${ }^{19-21}$ for the preparation of H-phosphonate monoesters. ${ }^{31} \mathrm{P}$ NMR studies shown that also H-phosphonate diesters can be efficiently formed under the Mitsunobu coupling conditions.

Further synthetic and mechanistic studies on the Mitsunobu reaction involving $\mathrm{H}-$ phosphonates as acidic pronucleophiles are in progress in this Laboratory.

\section{Experimental Section}

General Procedures. Pyridine was distilled from $\mathrm{CaH}_{2}$ and stored over molecular sieves $4 \AA$. THF was distilled directly before the use from sodium/benzophenone. $\mathrm{CH}_{2} \mathrm{Cl}_{2}$ was distilled directly before the use from $\mathrm{P}_{2} \mathrm{O}_{5}$. Phosphonic acid and pivaloyl chlorides were commercial grades from Aldrich. A suitably protected nucleosides 1a-d were prepared analogously to the published procedures. ${ }^{22}$

Progress of the reactions was monitored by thin layer chromatography (TLC) using silica gelcoated plates with a fluorescent indicator (Merck, Silica gel 60) and chloroform: methanol (9:1, $\mathrm{v} / \mathrm{v}$ ) as an eluent. Column chromatography was performed on silica gel (Grace Davison, Davsil, 0.035-0.070 mm). After chromatography, the fractions containing the desired products were pooled, evaporated, and dried under vacuum for 12 hours. ${ }^{1} \mathrm{H}-,{ }^{13} \mathrm{C}-$, and ${ }^{31} \mathrm{P}$ NMR spectra were recorded on Bruker Avance $400 \mathrm{MHz}$ instrument. Chemical shifts are reported in ppm, relative to TMS $\left({ }^{1} \mathrm{H}-,{ }^{13} \mathrm{C}-\mathrm{NMR}\right)$ and $85 \%$ aq. $\mathrm{H}_{3} \mathrm{PO}_{4}\left({ }^{31} \mathrm{P}\right.$ NMR). High resolution mass spectra (HRMS) were recorded on Bruker MicrOTOF ESI-TOF mass spectrometer.

\section{A general procedure for the preparation of nucleoside 5'-H-phosphonates 2a-d}

To a solution of a suitably protected nucleoside 1a-d $(0.5 \mathrm{mmol})$ in pyridine $(2 \mathrm{~mL})$ was added phosphonic acid $(1 \mathrm{mmol})$. The reaction mixture was made anhydrous by repeated evaporation of the added pyridine $(3 \times 2 \mathrm{~mL})$ and the residue was dissolved in the same solvent $(10 \mathrm{~mL})$. After addition of triphenylphosphine $(1 \mathrm{mmol})$ and diethyl azodicarboxylate $(1 \mathrm{mmol})$ the reaction mixture was stirred until complete disappearance of the starting material 1 (ca 20-30 min, TLC analysis). The solvent was removed under reduced pressure, the residue dissolved in dichloromethane, and purified by a silica gel column chromatography (a stepwise gradient of methanol and triethylamine in dichloromethane, $0-10 \%$ and $0.02-0.5 \%$, respectively). Compounds 2a-d (purity $>98 \%,{ }^{1} \mathrm{H}$ NMR spectroscopy) were obtained as white, amorphous solids.

3'-O-(4,4-Dimethoxytrityl)thymidin-5'-yl H-phosphonate, triethylammonium salt (2a).Yield: $302 \mathrm{mg}, 85 \% .{ }^{1} \mathrm{H}$ NMR $\left(400 \mathrm{MHz}, \mathrm{CDCl}_{3}\right): \delta 7.71(\mathrm{~s}, \mathrm{H} 6,1 \mathrm{H}), 7.46-7.14$ (m, $\mathrm{Ar}_{\mathrm{DMT}}$,

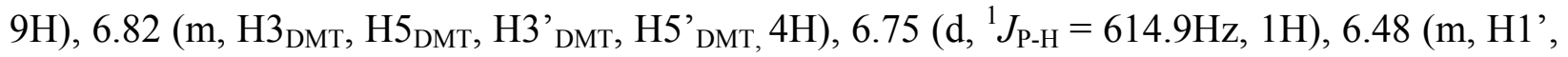
1H), 4.37 (m, H3', 1H), 3.89 (m, H4', 1H), 3.85-3.76 (m, 2×OCH, $\mathrm{H} 5$ ', 7H), 3.52 (m, H5', 1H), $2.96\left(\mathrm{q},{ }^{3} \mathrm{~J}=7.3 \mathrm{~Hz}, \mathrm{~N}\left(\mathrm{CH}_{2} \mathrm{CH}_{3}\right)_{3}, 6 \mathrm{H}\right), 1.91\left(\mathrm{~s}, 5 \mathrm{CH}_{3}, 3 \mathrm{H}\right), 1.82\left(\mathrm{~m}, \mathrm{H} 2\right.$ ', H2', 2H), 1.24 (t, ${ }^{3} J=$ $\left.7.3 \mathrm{~Hz}, \mathrm{~N}\left(\mathrm{CH}_{2} \mathrm{CH}_{3}\right)_{3}, 9 \mathrm{H}\right) .{ }^{13} \mathrm{C} \mathrm{NMR}\left(100 \mathrm{MHz}, \mathrm{CDCl}_{3}\right): \delta 164.1(\mathrm{~s}), 158.8(\mathrm{~s}), 150.8(\mathrm{~s}), 145.3$ 
(s), 136.7 (s), 136.5 (d), 130.4 (d), 128.5 (d), 128.2 (d), 127.2 (d), 113.52 (d), 113.50 (d), 111.3 (d), 87.4 (d), 85.5 (d, ${ }^{3} J_{\mathrm{POCC}}=8.1 \mathrm{~Hz}, \mathrm{C} 4$ '), 85.0 (s), 75.5 (d), 63.8 (d, ${ }^{2} J_{\mathrm{POC}}=4.4 \mathrm{~Hz}, \mathrm{C} 5$ '), 55.4 (q), $45.6(\mathrm{t}), 39.6$ (t), 12.6 (q), 8.8 (q). ${ }^{31} \mathrm{P}$ NMR (162 MHz, $\left.\mathrm{CDCl}_{3}\right): \delta=4.78 \mathrm{ppm}\left({ }^{1} J_{\mathrm{PH}}=615\right.$ Hz). HRMS: $m / z$ 607.1821 ([M-TEAH] $]^{-} \mathrm{C}_{31} \mathrm{H}_{32} \mathrm{~N}_{2} \mathrm{O}_{9} \mathrm{P}^{-}$calcd. 607.1851).

3'-O-(4,4-Dimethoxytrityl)- $\mathrm{N}^{\mathbf{4}}$-benzoyldeoxycytidin-5'-yl H-phosphonate, triethylammonium salt , triethylammonium salt(2b). Yield: $336 \mathrm{mg}, 84 \% .{ }^{1} \mathrm{H}$ NMR (400 MHz, $\left.\mathrm{CDCl}_{3}\right): \delta 8.44\left(\mathrm{~d},{ }^{3} J=7.4 \mathrm{~Hz}, \mathrm{H} 3,1 \mathrm{H}\right), 7.88\left(\mathrm{~m}, \mathrm{H} 2_{\mathrm{Bz}}, \mathrm{H} 6_{\mathrm{Bz}}, 2 \mathrm{H}\right), 7.60-7.19$ (m, DMT, Bz, H4,

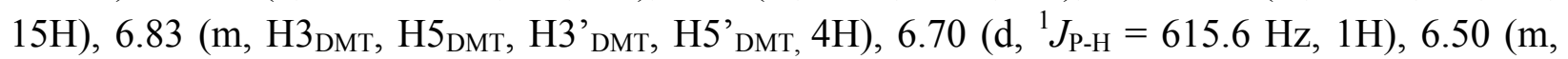
H1', 1H), 4.37 (m, H3', 1H), 3.78 (m, 2× $\mathrm{OCH}_{3}, \mathrm{H} 5$ ', 7H), 3.76 (m, H4', 1H), 3.36 (m, H5', 1H), 3.02 (q, $\left.{ }^{3} J=7.3 \mathrm{~Hz}, \mathrm{~N}\left(\mathrm{CH}_{2} \mathrm{CH}_{3}\right)_{3}, 6 \mathrm{H}\right), 2.43$ (m, H2”, $\left.1 \mathrm{H}\right), 1.87$ (m, H2', $\left.1 \mathrm{H}\right), 1.28$ (t, ${ }^{3} J=7.3$ $\left.\mathrm{Hz}, \mathrm{N}\left(\mathrm{CH}_{2} \mathrm{CH}_{3}\right)_{3}, 9 \mathrm{H}\right) .{ }^{13} \mathrm{C} \mathrm{NMR}\left(100 \mathrm{MHz}, \mathrm{CDCl}_{3}\right): \delta 162.0(\mathrm{~s}), 158.7$ (s), 145.7(s), 145.2(s), 136.5 (s), 136.4 (s), 133.1 (d), 130.31 (d), 130.26 (d), 129.0 (d), 128.4 (d), 128.0 (d), 127.6 (d), 127.1 (d), 113.4 (d), 96.6, 87.7, 87.3 (s), 86.3 (d, ${ }^{3} J_{\mathrm{POCC}}=8.0 \mathrm{~Hz}, \mathrm{C} 4$ ') $75.3,63.4$ (d, ${ }^{3} J_{\mathrm{POC}}=3.9$ $\mathrm{Hz}, \mathrm{C}^{\prime}$ '), 55.3 (q), 45.5 (t), 41.4 (t), 8.6 (q). ${ }^{31} \mathrm{P} \mathrm{NMR}\left(162 \mathrm{MHz}, \mathrm{CDCl}_{3}\right): \delta=4.57 \mathrm{ppm}\left({ }^{1} J_{\mathrm{PH}}=\right.$ $616 \mathrm{~Hz}$ ). HRMS: $m / z 696.2143$ ([M-TEAH] $]^{-} \mathrm{C}_{37} \mathrm{H}_{35} \mathrm{~N}_{3} \mathrm{O}_{9} \mathrm{P}^{-}$calcd. 696.2116).

3'-O-(4,4-Dimethoxytrityl))- $N^{\mathbf{6}}$-benzoyldeoxyadenosin-5'-yl H-phosphonate, triethylammonium salt (2c). Yield: $304 \mathrm{mg}, 74 \% .{ }^{1} \mathrm{H} \mathrm{NMR}\left(400 \mathrm{MHz}, \mathrm{CDCl}_{3}\right): \delta 9.14$ (bs, N6H, 1H), 8.80 and $8.70(2 \times \mathrm{s}, \mathrm{H} 2$ and $\mathrm{H} 8,2 \mathrm{H}), 8.01\left(\mathrm{~m}, \mathrm{H} 2_{\mathrm{Bz}}, \mathrm{H} 6_{\mathrm{Bz}}, 2 \mathrm{H}\right), 7.62-7.20$ (m, DMT, Bz, 13H), 6.85 (m, H3 ${ }_{\text {DMT }}, \mathrm{H}^{\mathrm{DMT}}$, H3 ' $\left.{ }_{\mathrm{DMT}}, \mathrm{H} 5^{\prime}{ }_{\mathrm{DMT}}, 4 \mathrm{H}\right), 6.74$ (d, $\left.{ }^{1} J=616.9 \mathrm{~Hz}, \mathrm{P}-\mathrm{H}, 1 \mathrm{H}\right), 6.70$ (m, H1', 1H), 4.52 (m, H3', 1H), 3.94 (m, H4', 1H), 3.86-3.77 (m, H5', 2× $\left.\mathrm{OCH}_{3}, 7 \mathrm{H}\right), 3.53$ (m, H5”, 1H), 2.99 (q, $\left.{ }^{3} J=7.3 \mathrm{~Hz}, \mathrm{~N}\left(\mathrm{CH}_{2} \mathrm{CH}_{3}\right)_{3}, 6 \mathrm{H}\right), 2.44$ (m, H2', 1H), 2.11 (m, H2”, 1H), 1.23 (t, ${ }^{3} J=7.3$ $\left.\mathrm{Hz}, \mathrm{N}\left(\mathrm{CH}_{2} \mathrm{CH}_{3}\right)_{3}\right) .{ }^{13} \mathrm{C} \mathrm{NMR}\left(100 \mathrm{MHz}, \mathrm{CDCl}_{3}\right): \delta 165.1$ (s), 159.0 (s), 152.7 (s), 152.3 (s), 149.5 (s), $145.3(\mathrm{~s}), 142.6$ (d), 136.5 (s), $134.1(\mathrm{~s}), 132.9$ (d), 130.47 (d), 130.45 (d), 129.1 (d), 128.5 (d), 128.3 (d), 128.1 (d), 127.3 (d), 123.2 (d), 113.6 (d), 87.6 (s), 86.3 (d, ${ }^{3} J_{\text {POCC }}=7.4 \mathrm{~Hz}$, C4'), 84.5 (d), 75.8 (d), 63.9 (d, ${ }^{2} J_{\mathrm{POC}}=4.3 \mathrm{~Hz}, \mathrm{C} 5$ '), 55.5 (q), 45.6 (t), 40.8 (t), 8.8 (q). ${ }^{31} \mathrm{P}$ NMR $\left(162 \mathrm{MHz}, \mathrm{CDCl}_{3}\right): \delta=4.70 \mathrm{ppm}\left({ }^{1} J_{\mathrm{PH}}=618 \mathrm{~Hz}\right)$. HRMS: $m / z 720.2264$ ([M-TEAH]', $\mathrm{C}_{38} \mathrm{H}_{35} \mathrm{~N}_{5} \mathrm{O}_{8} \mathrm{P}^{-}$calcd. 720.2229).

3'-O-(4,4-Dimethoxytrityl)- $\mathrm{N}^{\mathbf{2}}$-isobutyryldeoxyguanosin-5'-yl H-phosphonate, triethylammonium salt (2d). Yield: $338 \mathrm{mg}, 84 \% .{ }^{1} \mathrm{H}$ NMR (400 MHz, $\left.\mathrm{CDCl}_{3}\right): \delta 12.7(\mathrm{~s}, \mathrm{NH}, 1 \mathrm{H})$, 12.3 (s, NH, 1H), 7.64 (s, H8, 1H), 7.45-7.17 (m, DMT, 9H), 6.80 (m, H3 ${ }_{\mathrm{DMT}}, \mathrm{H} 5_{\mathrm{DMT}}, \mathrm{H} 3{ }^{\text {DMT }}$, H5 ' $\left.{ }_{\text {DMT, }} 4 \mathrm{H}\right), 6.71$ (d, ' $\left.J_{\mathrm{P}-\mathrm{H}}=620.0 \mathrm{~Hz}, 1 \mathrm{H}\right), 6.15$ (m, H1', 1H), 4.34 (m, H3', 1H), 3.91 (m, H4', $1 \mathrm{H}), 3.85$ (m, H5', 1H), $3.75\left(\mathrm{~s}, 2 \times \mathrm{OCH}_{3}, 6 \mathrm{H}\right), 3.67$ (m, H5”, 1H), 2.96-2.82 (m, $\left(\mathrm{CH}_{3}\right)_{2} \mathrm{CHibu}$, $\mathrm{N}\left(\mathrm{CH}_{2} \mathrm{CH}_{3}\right)_{3}, \mathrm{H} 2$ ', $\left.8 \mathrm{H}\right), 1.64(\mathrm{~m}, \mathrm{H} 2$ ', $1 \mathrm{H}), 1.17-1.11\left(\mathrm{~m},\left(\mathrm{CH}_{3}\right)_{2} \mathrm{CHibu}, \mathrm{N}\left(\mathrm{CH}_{2} \mathrm{CH}_{3}\right)_{3}, 15 \mathrm{H}\right) .{ }^{13} \mathrm{C}$ NMR (100 MHz, $\mathrm{CDCl}_{3}$ ): $\delta 181.0$ (s), 158.7 (s), 155.9 (s), 148.5 (s), 148.1 (s), 145.0 (s), 139.1 (d), 136.1 (s), 130.1(d), 128.1 (d), 128.0 (d), 127.0 (d), 122.8 (d), 113.3 (d), 87.5 (s), 87.3 (d), $85.7\left(\mathrm{~d},{ }^{3} J_{\text {POCC }}=8.4 \mathrm{~Hz}, \mathrm{C} 4\right.$ '), 75.0 (d), 63.9 (d, ${ }^{2} J_{\text {POC }}=3.8 \mathrm{~Hz}, \mathrm{C} 5$ '), 55.2 (q), 45.4 (q), 37.7 (t), 35.2 (d), 19.3 (q), 18.9 (q), 8.5 (q). ${ }^{31} \mathrm{P} \mathrm{NMR}$ (162 MHz, $\left.\mathrm{CDCl}_{3}\right): \delta=4.93 \mathrm{ppm}\left({ }^{1} J_{\mathrm{PH}}=622 \mathrm{~Hz}\right)$. HRMS: $m / z$ 702.2320 ([M-TEAH] $]^{-}, \mathrm{C}_{35} \mathrm{H}_{37} \mathrm{~N}_{5} \mathrm{O}_{9} \mathrm{P}^{-}$calcd. 702.2334). 


\section{Acknowledgements}

Financial support from the Swedish Research Council is gratefully acknowledged.

\section{References and Notes}

1. (a) Mitsunobu, O. Synthesis 1981, 1. (b) But, T. Y. S.; Toy, P. H. Chem. Asian J. 2007, 2, 1340.

2. But, T. Y. S.; Toy, P. H. J. Am. Chem. Soc. 2006, 128, 9636.

3. (a) Lipshutz, B. H.; Chung, D. W.; Rich, B.; Corral, R. Org. Lett. 2006, 8, 5069. (b) Veliz, E. A.; Beal, P. A. Tetrahedron Lett. 2006, 47, 3153. (c) Mahdavi, H.; Amani, J. Tetrahedron Lett. 2008, 49, 2204. (d) Dandapani, S.; Curran, D. P. J. Org. Chem. 2004, 69, 8751. (d) Elson, K. E.; Jenkins, I. D.; Loughlin, W. A. Tetrahedron Letters 2004, 45, 2491. (e) Fairfull-Smith, K. E.; Jenkins, I. D.; Loughlin, W. A. Org. Biomol. Chem. 2004, 2, 1979.

4. (a) Dandapani, S.; Curran, D. P. Chem. Eur. J. 2004, 3130. (b) Dembinski, R. Eur. J. Org. Chem. 2004, 2763.

5. Proctor, A. J.; Beautement, K.; Clough, J. M.; Knighta, D. W.; Li, Y. Tetrahedron Lett. 2006, $47,5151$.

6. Stawinski, J.; Kraszewski, A. Acc. Chem. Res. 2002, 35, 952.

7. Kraszewski, A.; Stawinski, J. Pure \& Appl. Chem. 2007, 79, 2217.

8. (a) Hayakawa, Y.; Hirabayashi, Y.; Hyodo, M.; Yamashita, S.; Matsunami, T.; Cui, D. M.; Kawai, R.; Kodama, H. Eur. J. Org. Chem. 2006, 3834. (b) Almer, H.; Szabo, T.; Stawinski, J. Chem. Commun. 2004, 290.

9. (a) Grochowski, E.; Hilton, B. D.; Kupper, R. J.; Michejda, C. J. Am. Chem. Soc. 1982, 104, 6876. (b) Varasi, M.; Walker, K. A. M.; Maddox, M. L. J. Org. Chem. 1987, 52, 4235. (c) Hughes, D. L.; Reamer, R. A.; Bergan, J. J.; Grabowski, E. J. J. J. Am. Chem. Soc. 1988, 110, 6487. (d) Camp, D.; Jenkins, I. D. J. Org. Chem. 1989, 54, 3045. (e) Camp, D.; Jenkins, I. D. J. Org. Chem. 1989, 54, 3049. (f) Crich, D.; Dyker, H.; Harris, R. J. J. Org. Chem. 1989, 54, 257. (f) Campbell, D. A. J. Org. Chem. 1992, 57, 6331. (g) McNulty, J.; Capretta, A.; Laritchev, V.; Dyck, J.; Robertson, A. J. Ang. Chem. Int. Ed. Eng. 2003, 42, 4051.

10. Ahn, C. J.; Correia, R.; DeShong, P. J. Org. Chem. 2002, 67, 1751.

11. (a) Morrison, D. J. Org. Chem. 1958, 23, 1072. (b) Brunn, E.; Huisgen, R. Ang. Chem. Int. Ed. 1969, 8, 513.

12. Hughes, D. L.; Reamer, R. A. J. Org. Chem. 1996, 61, 2967.

13. Schenk, S.; Weston, J.; Anders, E. J. Am. Chem. Soc. 2005, 127, 12566.

14. (a) Mitsunobu, O.; Kato, K.; Kimura, J. J. Am. Chem. Soc. 1969, 91, 6510. (b) Mitsunobu, O.; Eguchi, M. Bull. Chem. Soc. Jpn 1971, 44, 3427.

15. Kimura, J.; Fujisawa, Y.; Yoshizawa, T.; Fukuda, K.; Mitsunobu, O. Bull. Chem. Soc. Jpn 1979, 52, 1191. 
16. (a) Saady, M.; Lebeau, L.; Mioskowski, C. Tetrahedron Lett. 1995, 36, 2239. (b) Saady, M.; Lebeau, L.; Mioskowski, C. Synlett 1995, 643.

17. Jahn, W. Chem. Ber. 1965, 98, 1705.

18. (a) Dodge, J. A.; Trujillo, J. I.; Presnell, M. J. Org. Chem. 1994, 59, 234. (b) Harvey, P. J.; von Itzstein, M.; Jenkins, I. D. Tetrahedron 1997, 53, 3933.

19. Stawinski, J.; Thelin, M. Nucleosides Nucleotides 1990, 9, 129.

20. Stawinski, J. Some Aspects of H-Phosphonate Chemistry. In Handbook of Organophosphorus Chemistry; R. Engel, Ed.; Marcel Dekker: New York, 1992; p. 377.

21. (a) Jankowska, J.; Sobkowski, M.; Stawinski, J.; Kraszewski, A. Tetrahedron Lett. 1994, 35, 3355. (b) Stawinski, J.; Strömberg, R. Di- and oligonucleotide Synthesis Using HPhosphonate Chemistry. In Oligonucleotide Synthesis: Methods and Applications; P. Herdewijn, Ed.; Humana Press: Totowa, NJ, 2004; Vol. 288, p. 81.

22. Gait, M. J. Ed. Oligonucleotide synthesis. A practical approach; IRL Press: Oxford, 1984; p. 23. 